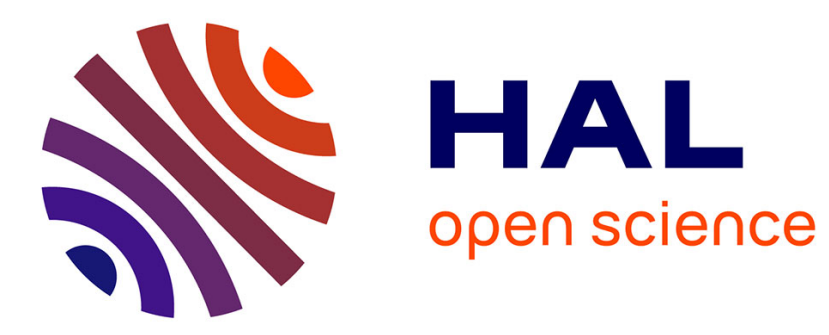

\title{
On a differential algebraic system structure theory
}

\author{
Sette Diop, Youcef Aït Amirat
}

\section{To cite this version:}

Sette Diop, Youcef Ait Amirat. On a differential algebraic system structure theory. IFAC 2013 - 5th Symposium on System Structure and Control, Feb 2013, Grenoble, France. 10.3166/ejc.12.545-558 . hal-00828624

\section{HAL Id: hal-00828624 \\ https://hal-centralesupelec.archives-ouvertes.fr/hal-00828624}

Submitted on 31 May 2013

HAL is a multi-disciplinary open access archive for the deposit and dissemination of scientific research documents, whether they are published or not. The documents may come from teaching and research institutions in France or abroad, or from public or private research centers.
L'archive ouverte pluridisciplinaire HAL, est destinée au dépôt et à la diffusion de documents scientifiques de niveau recherche, publiés ou non, émanant des établissements d'enseignement et de recherche français ou étrangers, des laboratoires publics ou privés. 


\title{
On a differential algebraic system structure theory
}

\author{
S. Diop * Y. Aït-Amirat ${ }^{* *}$ \\ * Labratoire des Signaux et Systèmes, CNRS - Supélec - Univ. Paris \\ Sud, Plateau de Moulon, 91192 Gif sur Yvette cedex France (e-mail: \\ diop@lss.supelec.fr). \\ ** Femto-ST, UMR CNRS 6174 / Univ. Franche-Comté - FC LAB \\ Rue Thierry Mieg, 90010 Belfort cedex France (e-mail: \\ youcef.ait-amirat@univ-fcomte.fr)
}

\begin{abstract}
The structure at infinity and the essential structure are two control theory notions which were first defined for linear, then for the so-called affine, systems. And they were shown to be useful tools for the study of the fundamental problem of noninteracting control. They also appeared as related to the solutions of other important control problems such as disturbance decoupling. Their definitions are however entirely in terms of an algorithm, namely the so-called structure algorithm. The present work proposes new definitions with some advantages: they extend the class of systems from linear and affine systems to systems which may be described by algebraic differential equations, they are not tied to specific algorithms, and finally they provide more information on system structure. Let a system be a set of differential equations in variables which are grouped as $m$ inputs, $p$ outputs and $n$ latent variables. To each input component is attached a rational integer, which, for a single input single output system defined by a single differential equation, is the difference between the order in the output and the order in the input of the differential equation defining the system. The $m$-tuple of these rational integers is the new structure at infinity of the system. Associated to the structure at infinity is also defined a $p$-tuple of rational integers representing a new notion of essential structure. The old structure at infinity is shown to be recoverable from the new one. Computations of system structure based upon the suggested definitions are quite complex. The present paper focuses on proofs of algorithms which attempt to reduce the complexity of these computations.
\end{abstract}

Keywords: Structure at infinity; Essential structure; Non-interacting control; Nonlinear system theory; Algebraic system theory

\section{INTRODUCTION}

The structure at infinity and the essential structure are two control theory notions which were first defined for linear, then for the so-called affine, systems. And they were shown to be useful tools for the study of the fundamental problem of noninteracting control. They also appeared as related to the solutions of other important control problems such as disturbance decoupling. The reader is referred to the numerous papers in the literature, for example di Benedetto et al. (1989); Aït Amirat (1994); Belur (2011); Laakkonen and Pohjolainen (2011); Pereira da Silva et al. (2006).

For an affine control system

$$
\left\{\begin{array}{l}
\dot{x}=A(x)+\sum_{i=1}^{m} u_{i} B_{i}(x), \\
y=C(x),
\end{array}\right.
$$

referring to di Benedetto et al. (1989), the structure at infinity was defined from a finite sequence of natural integers $\rho_{1}, \rho_{2}, \ldots, \rho_{n}$ where $n$ is the number of state components in equation (1), and $\rho_{i}$ is obtained through the so-called structure algorithm (see $\S 5$ for details). Firstly, such a definition is algorithmic in the sense that the $\rho$ 's are ultimately defined through the structure algorithm. Secondly, the definition is valid for affine control systems, only.

The goal in this work is

- to propose such a nonalgorithmic definition of the structure at infinity,

- which fits for a larger class of systems such as systems given by input output description, and those given by general implicit algebraic differential equations with latent variable,

- to propose a constructive method for the computation of the structure at infinity,

- and, finally, to investigate the basic properties of systems which might be clarified through their structures at infinity.

For that, techniques stemming from differential algebra, as first introduced in control theory by Fliess (1989), are used. This primary issue may be said to have been achieved. The way leading to the new definitions may be found a long drive, due to the very detailed aspects of a system that are captured by its structure at infinity. Corroborating this matter of fact is the uneasy situation 
that only a poor intuitive notion of what the structure at infinity can be expressed in more down to earth words.

The structure at infinity may be thought of as a (signed) distance of output components from the input components, this intuitive distance idea being evaluated in terms of numbers of integrators from the input components to the output components. A negative number there corresponds to derivatives instead of integrators.

At this stage it is better to use simple specific examples. The structure at infinity of

$$
\left\{\begin{array}{l}
\ddot{y}_{1}+y_{1}=u_{1}, \\
\ddot{y}_{1}+y_{2}=u_{2}
\end{array}\right.
$$

is the following two couples of integers: $(2,0),(0,2)$. The multiplicity of the structure at infinity reflects the arbitrariness of which input component is looked first. Actually, it may be said that from $u_{1}$ to the output components there are two integrators; and then $u_{2}$ is given by $u_{2}=u_{1}+y_{2}-y_{1}$. But it may equally be said that from $u_{2}$ to the output components there are two integrators; and then $u_{1}$ is given by $u_{1}=u_{2}+y_{1}-y_{2}$.

Again, instead of the list consisting of the single integer 1, the structure at infinity of

$$
\dot{y}=u_{1}+u_{2}
$$

is the following two couples $(1, \varpi),(\varpi, 1)$, where $\varpi$ is merely a symbol which indicates, first, the noninvertibility of system (3), and second, the arbitrariness of the choice between $u_{1}$ and $u_{2}$ when one thinks to the minimum number of integrators from the input components to the output components. This point becomes clearer through the following rather more elaborated example

$$
\left\{\begin{array}{l}
\ddot{y}_{1}+y_{1}=u_{1}+\dot{u}_{2}+u_{3} \\
\ddot{y}_{2}=\ddot{u}_{1}
\end{array}\right.
$$

the structure at infinity of this system, along the same beliefs, is the single triplet $(0,1, \varpi)$.

The viewpoint is thus that the structure at infinity is a collection of $m$-tuples of rational integers and a phantom. The trick of a phantom is used to carry over an information that is essential and consists in, if $\sigma=\left(\sigma_{1}, \ldots, \sigma_{m}\right)$ is proclaimed a structure at infinity of a system then the correspondence between the integers composing $\sigma$ and the input components of the system is made transparent. The phantom is used to specify the input components which are chosen as indeterminates. Such a precise definition of the structure at infinity makes the concept relative to the choice of the order in which the input components are considered. Actually, the definition relies on the choice of a differential transcendence base of the external field (the differential field attached to the external behavior of the system) among the components of the input and output. This is achieved through renumberings of the input and output components; renumbering named differential input output base (bio for short, and bios for the plural). A bio is thus a renumbering $\mathfrak{B}=\left(u_{i_{1}}, \ldots, u_{i_{m}}, y_{j_{1}}, \ldots, y_{j_{p}}\right)$ of $\left(u_{1}, \ldots, u_{m}, y_{1}, \ldots, y_{p}\right)$ where $u=\left(u_{1}, \ldots, u_{m}\right)$, and $y=\left(y_{1}, \ldots, y_{p}\right)$ are the input, and output, respectively, $\rho$ is the differential output rank (see Definition 4) of the system; the last $m-\rho$ components of the input together with the first $\rho$ components of the output are assumed to form a differential transcendence base of the external field. Now, $u_{i_{\rho}}$ satisfies a unique (up to the multiplication by an element of the base field of coefficients of the system) differential polynomial with coefficients depending only on $\left(y_{j_{1}}, \ldots, y_{j_{\rho}}, u_{i_{\rho+1}}, \ldots, u_{i_{m}}\right)$, and of minimal order in $u_{i_{\rho}}$. This defines a unique integer, the difference between the order in $y$ of this differential equation and its order in $u_{i_{\rho}}$. This is the first step of the construction of a $\rho$-tuple of integers attached to any given bio, $\mathfrak{B}$. The structure at infinity of the system is the minimum of all those $\rho$-tuples according to some well ordering. Actually, what is thus defined is the canonical structure at infinity. While any given system has one and only one canonical structure at infinity, its structure at infinity is a list of $m$ tuples of integers and a phantom element denoted by $\varpi$. The elements of the $m$-tuples which are $\varpi$ correspond to $u_{i_{\rho+1}}, \ldots, u_{i_{m}}$.

The essential structure is defined correspondingly to the same bio.

The rest of the paper is organized as follows. In the next section are collected materials needed in $\S 3$ where the new definitions are given. Then follow 2 sections which are dedicated to the computing algorithms and the verification that the new definitions allow recovery of old ones.

\section{PRELIMINARIES}

Notions of differential algebra are not recalled in this paper. The reader who is not familiar with them is referred to Kolchin's book Kolchin (1973). An account of basic facts has been appended to Diop (2002). Differential fields are always assumed ordinary ones (that is, with one and only one derivation) and with characteristic zero.

Given a field extension $\mathbf{K}$ of $\mathbf{k}$, the transcendence degree of $\mathbf{K}$ over $\mathbf{k}$ is denoted by $\mathrm{d}_{\mathbf{k}}^{\circ} \mathbf{K}$. If $\mathbf{K}$ is a differential field extension of $\mathbf{k}, \partial_{\mathbf{k}}^{\circ} \mathbf{K}$ designates the differential transcendence degree of $\mathbf{K}$ over $\mathbf{k}$.

Lemma 1. Given a differential transcendence base $\tau=$ $\left(\tau_{1}, \ldots, \tau_{\mu}\right)$ of a differential field extension $\mathbf{K}$ over $\mathbf{k}$, and given an element $\xi$ of $\mathbf{K}$, there is a $\mathbf{k}$-irreducible differential polynomial in $\mathbf{k}\left\{T_{1}, \ldots, T_{\mu}, X\right\}$ which is annulled by $\left(\tau_{1}, \ldots, \tau_{\mu}, \xi\right)$, and which is with minimal order and minimal degree in $X$. Such a polynomial is unique in $\mathbf{k}\left\{T_{1}, \ldots, T_{\mu}, X\right\}$ up to the multiplication by an element of $\mathbf{k}$.

Proof. Omitted for lack of space.

Definition 2. The "unique" differential polynomial of Lemma 1 is called the differential minimal polynomial of $\xi$ over $\mathbf{k}\langle\tau\rangle$.

The differential minimal polynomial of $\xi$ over $\mathbf{k}\langle\tau\rangle$ is not uniquely defined in $\mathbf{k}\left\{T_{1}, \ldots, T_{\mu}, X\right\}$ if $\tau$ is not a differential transcendence base of $\mathbf{k}\langle\tau, \xi\rangle$ over $\mathbf{k}$.

Lemma 3. Let $\mathbf{K}$ be a differential field extension of $\mathbf{k}$. Let $\tau=\left(\tau_{1}, \ldots, \tau_{\mu}\right)$ be a differential transcendence base of $\mathbf{K}$ over $\mathbf{k}$. Let $\xi$ be an element of $\mathbf{K}$. Let $\mathfrak{m}(\xi)$ be the differential minimal polynomial of $\xi$ in $\mathbf{k}\left\{T_{1}, \ldots, T_{\mu}, X\right\}$ over $\mathbf{k}\langle\tau\rangle$. If $\mathfrak{m}(\xi)$ effectively depends on $\tau_{i_{0}}$ then $\tau_{1}, \ldots, \tau_{i_{0}-1}, \xi, \tau_{i_{0}+1}, \ldots, \tau_{\mu}$ are differentially algebraically independent over $\mathbf{k}$.

Proof. Omitted for lack of space. 
In this approach a (differential) (algebraic) system with $s$ variables, and with coefficients in $\mathbf{k}$ is a differential affine variety $\mathcal{X} \subseteq \overline{\mathbf{k}}^{s}$ defined over $\mathbf{k}$ where $\overline{\mathbf{k}}$ is a differential closure of $\mathbf{k}$. The differential dimension of $\mathcal{X}$ over $\mathbf{k}$ is the (minimum) number of input components. An input is a differential transcendence basis of $\mathbf{k}\langle\mathcal{X}\rangle$ over $\mathbf{k}$. Once an input $u$ is chosen the other components of the system variable are differentially algebraic over $\mathbf{k}\langle u\rangle$.

The system variables are partioned into the input $u=$ $u_{1}, \ldots, u_{m}$, the output $y=y_{1}, \ldots, y_{p}$, and the latent variable $z=z_{1}, \ldots, z_{n}$. The input $u$ often reduces to the control. The variable $y$ is the online measurements, and $z$ is the remaining variables which are invoked in the description of the system but which are part neither of the input nor of the output. As known such a system is equivalently given by a set of differential polynomials $P(U, Z, Y)$ in $\mathbf{k}\{U, Z, Y\}$ where $U=U_{1}, \ldots, U_{m} Z=Z_{1}, \ldots, Z_{n}$ and $Y=Y_{1}, \ldots, Y_{p}$ are differential indeterminates over k. Since systems are assumed to be differential varieties the corresponding defining sets of differential polynomials are consequently assumed to generate differential prime ideals. The differential algebra, quotient of $\mathbf{k}\{U, Z, Y\}$ by the defining differential prime ideal of the system, is denoted by $\mathbf{k}\{\mathcal{X}\}$ or $\mathbf{k}\{u, z, y\}$. Its differential quotient field is denoted by $\mathbf{k}\langle u, z, y\rangle$. The ubiquitous class of control systems

$$
\begin{cases}\dot{x}_{i}=p_{i}(u, x) & (1 \leq i \leq n) \\ y_{j}=q_{j}(u, x) & (1 \leq j \leq p)\end{cases}
$$

are examples of differential algebraic systems according to the previous definition.

It may be interesting to consider more general differential algebraic systems by allowing not only implicit differential algebraic equations but differential algebraic inequations also. It also may be important to extend studies to systems where the input does not represent a differential transcendence base but merely a set of differential algebraic generators of $\mathbf{k}\{u, z, y\}$ over $\mathbf{k}$. These potential extensions are not considered in the present work.

Definition 4. Let $\mathcal{X}$ be a system, the transcendence degree of $\mathbf{k}\langle y\rangle$ over $\mathbf{k}$ is called the differential output rank of $\mathcal{X}$ and denoted by $\rho$.

Lemma 5. The differential output rank, $\rho$, of $\mathcal{X}$ is equal to $m-\partial_{\mathbf{k}\langle y\rangle}^{\circ} \mathbf{k}\langle u, y\rangle$ (which, in turn, is equal to $m-$ $\left.\partial_{\mathbf{k}\langle y\rangle}^{\circ} \mathbf{k}\langle u, z, y\rangle\right)$.

Proof. Omitted due to lack of space.

Definition 6. A differential base of input output (bio, for short, and bios in plural) of a system $\mathcal{X}$ with positive differential output rank $\rho$ is a renaming, $\left(u_{i_{1}}, \ldots, u_{i_{m}}, y_{j_{1}}, \ldots, y_{j_{p}}\right)$, of the external variables, $\mathrm{u}$ and $\mathrm{y}$, such that the $m-\rho$ last input components, $u_{i_{\rho+1}}, \ldots, u_{i_{m}}$, together with the $\rho$ first output components, $y_{j_{1}}, \ldots, y_{j_{\rho}}$, form a differential transcendence base of $\mathbf{k}\langle u, z, y\rangle$. The set of bios of a system with differential output rank zero is assumed to be the empty set. Throughout, unless otherwise stated, a bio $\mathfrak{B}$ will be denoted by $\mathfrak{B}=\left(\left(u_{i_{1}}, \ldots, u_{i_{\rho}}\right),\left(u_{i_{\rho+1}}, \ldots, u_{i_{m}}\right)\right.$, $\left.\left(y_{j_{1}}, \ldots, y_{j_{\rho}}\right),\left(y_{j_{\rho+1}}, \ldots, y_{j_{p}}\right)\right)$. When no confusion is to be feared the outmost parentheses will be dropped.
Let $\mathfrak{B}$ be a bio of $\mathcal{X}$. Let $l$ be an index, $1 \leq l \leq \rho$. By Lemma 3 , if the differential minimal polynomial of $u_{i_{l}}$ over $\mathbf{k}\left\langle y_{j_{1}}, \ldots, y_{j_{\rho}}, u_{i_{\rho+1}}, \ldots, u_{i_{m}}\right\rangle$ effectively depends on $y_{j_{l^{\prime}}}$ then $\left(y_{j_{1}}, \ldots, y_{{l^{\prime}-1}^{\prime}}, u_{i_{l}}, y_{{j^{\prime}+1}^{\prime}}, \ldots, y_{j_{\rho}}, u_{i_{\rho+1}}, \ldots, u_{i_{m}}\right)$ is a differential transcendence base of $\mathbf{k}\langle u, z, y\rangle$ over $\mathbf{k}$.

\section{THE NEW DEFINITIONS}

Definition 7. Let $P=P(U, Z, Y)$ be a differential polynomial in the differential polynomial algebra $\mathbf{k}\{U, Z, Y\}$. The input order of $P$ in $U_{l}$ is the order of $P$ in $U_{l}$ if $P$ effectively involves $U_{l}$, and if this component of the input appears in $P$ at order equal to the order of $P$ in $(U, Z)$; the input order of $P$ in $U_{l}$ is $\infty$ if $P$ effectively involves $U_{l}$ but at order lower than the order of $P$ in $(U, Z)$; and the input order of $P$ in $U_{l}$ is $-\infty$ if $P$ is free of $U_{l}$. The input order of $P$ in $U_{l}$ will be denoted by $\alpha_{P, l}$. The order of $P$ in a variable is assumed to be $-\infty$ if $P$ is free of that variable. $\beta_{P}$ will stand for the order of $P$ in $Y$. In what follows the ring $\mathbb{Z}$ of rational integers is extended by adjoining to it the two elements $-\infty$ and $\infty$. The natural order of integers is consequently prolonged by the usual assumption: $-\infty<n<\infty$, for any $n \in \mathbb{Z}$. Also, $-(-\infty)=\infty$, and $n+\infty=\infty$, and $n-\infty=-\infty$, for any $n \in \mathbb{Z}$, and $\infty+\infty=\infty$, and $-\infty-\infty=-\infty$. Note that $\infty-\infty$ and $-\infty+\infty$ are undefined. Finally, when the difference $\beta_{P}-\alpha_{P, l}$ is defined, it is denoted by $\sigma_{P, l}$.

Definition 8. Let $\mathfrak{B}$ be a bio of a system $\mathcal{X}$. For $l=$ $1,2, \ldots, \rho$, let $\mathfrak{m}_{l, i_{k}}$ denote the differential minimal polynomial of $u_{i_{k}}, 1 \leq k \leq \rho-l+1$, over the differential field $\mathbf{k}\left\langle y_{j_{1}}, \ldots, y_{j_{\rho-l+1}}, u_{i_{\rho-l+2}}, \ldots, u_{i_{m}}\right\rangle . \mathfrak{B}$ is said to be ordered if, for $l=1,2, \ldots, \rho, \sigma_{\mathfrak{m}_{l, i}-l+1}, i_{\rho-l+1}$ is lower than no other $\sigma_{\mathfrak{m}_{l, i_{k}}, i_{k}}, 1 \leq k \leq \rho-l+1$, and $y_{j_{\rho-l+1}}$ is one of the output components which appear in $\mathfrak{m}_{l, i_{\rho-l+1}}$ at order equal to the order of $\mathfrak{m}_{l, i_{\rho-l+1}}$ in $y$.

In the renaming of the input output components two things are of interest: on the one hand the differential transcendence base $\left(y_{j_{1}}, \ldots, y_{j_{\rho}}, u_{i_{\rho+1}}, \ldots, u_{i_{m}}\right)$, and on the other hand, the way $\left(y_{j_{1}}, \ldots, y_{j_{\rho}}\right)$ and $\left(u_{i_{1}}, \ldots, u_{i_{\rho}}\right)$ are numbered. The renumbering of $\left(y_{j_{\rho+1}}, \ldots, y_{j_{p}}\right)$, and $\left(u_{i_{\rho+1}}, \ldots, u_{i_{m}}\right)$ is really of no concern. This is the reason why the renaming $\left(u_{i_{1}}, \ldots, u_{i_{m}}, y_{j_{1}}, \ldots, y_{j_{p}}\right)$ of $\left(u_{1}, \ldots, u_{m}, y_{1}, \ldots, y_{p}\right)$ is qualified as a base. Any given bio can easily be renamed into an ordered one.

For instance, the differential output rank of system (2) is 2 , and its set of bios is

$$
\begin{aligned}
& \left(\left(u_{1}, u_{2}\right),(),\left(y_{1}, y_{2}\right),()\right), \quad\left(\left(u_{2}, u_{1}\right),(),\left(y_{1}, y_{2}\right),()\right), \\
& \left(\left(u_{1}, u_{2}\right),(),\left(y_{2}, y_{1}\right),()\right), \\
& \left(\left(u_{2}, u_{1}\right),(),\left(y_{2}, y_{1}\right),()\right) .
\end{aligned}
$$

Among these bios, only

$$
\left(\left(u_{1}, u_{2}\right),(),\left(y_{2}, y_{1}\right),()\right), \quad\left(\left(u_{2}, u_{1}\right),(),\left(y_{2}, y_{1}\right),()\right)
$$

are ordered ones.

The differential output rank of system (4) is 2 , and its set of bios is 
$\left(\left(u_{1}, u_{2}\right),\left(u_{3}\right),\left(y_{1}, y_{2}\right),()\right)$, $\left(\left(u_{3}, u_{1}\right),\left(u_{2}\right),\left(y_{1}, y_{2}\right),()\right)$, $\left(\left(u_{2}, u_{3}\right),\left(u_{1}\right),\left(y_{1}, y_{2}\right),()\right)$, $\left(\left(u_{1}, u_{3}\right),\left(u_{2}\right),\left(y_{1}, y_{2}\right),()\right)$, $\left(\left(u_{2}, u_{1}\right),\left(u_{3}\right),\left(y_{1}, y_{2}\right),()\right)$, $\left(\left(u_{3}, u_{2}\right),\left(u_{1}\right),\left(y_{1}, y_{2}\right),()\right)$,

$\left(\left(u_{1}, u_{2}\right),\left(u_{3}\right),\left(y_{2}, y_{1}\right),()\right)$ $\left(\left(u_{3}, u_{1}\right),\left(u_{2}\right),\left(y_{2}, y_{1}\right),()\right)$, $\left(\left(u_{2}, u_{3}\right),\left(u_{1}\right),\left(y_{2}, y_{1}\right),()\right)$, $\left(\left(u_{1}, u_{3}\right),\left(u_{2}\right),\left(y_{2}, y_{1}\right),()\right)$, $\left(\left(u_{2}, u_{1}\right),\left(u_{3}\right),\left(y_{2}, y_{1}\right),()\right)$, $\left(\left(u_{3}, u_{2}\right),\left(u_{1}\right),\left(y_{2}, y_{1}\right),()\right)$.

Among these bios, only

$\left(\left(u_{3}, u_{1}\right),\left(u_{2}\right),\left(y_{1}, y_{2}\right),()\right)$,

$\left(\left(u_{3}, u_{1}\right),\left(u_{2}\right),\left(y_{2}, y_{1}\right),()\right)$

$\left(\left(u_{1}, u_{2}\right),\left(u_{3}\right),\left(y_{2}, y_{1}\right),()\right)$,

are ordered ones.

Given a bio, $\left(u_{i_{1}}, \ldots, u_{i_{m}}, y_{j_{1}}, \ldots, y_{j_{p}}\right)$, for $l, 1 \leq l \leq \rho$, the notation $\mathfrak{m}_{l, i_{\rho-l+1}}$ will be shortened as $\mathfrak{m}_{i_{\rho-l+1}}$, and $\beta_{\mathfrak{m}_{i}{ }_{\rho-l+1}}$ as $\beta_{i_{\rho-l+1}}$, and $\alpha_{\mathfrak{m}_{i_{\rho-l+1}}, i_{\rho-l+1}}$ as $\alpha_{i_{\rho-l+1}}$, and $\sigma_{\mathfrak{m}_{i_{\rho-l+1}}, i_{\rho-l+1}}$ as $\sigma_{i_{\rho-l+1}}$. For each $l, 1 \leq l \leq \rho$, let $\gamma_{i_{k}}$ be the order in $y_{j_{\rho-l+1}}$ of the $\mathfrak{m}_{i_{k}}, 1 \leq k \leq \rho$, and let $\varepsilon_{j_{\rho-l+1}}$ be the maximum of the differences $\gamma_{i_{k}}-\alpha_{i_{k}}$. (Note that $\varepsilon_{j_{\rho-l+1}}$ is always defined.)

Definition 9. Ordering lexicographically the set of ordered bios of $\mathcal{X}$ according to their $\rho$-tuples $\left(\sigma_{i_{\rho}}, \sigma_{i_{\rho-1}}, \ldots, \sigma_{i_{1}}\right)$, a minimal bio of $\mathcal{X}$ is defined to be the least element of this set of ordered bios. The unique $\rho$-tuple $\left(\sigma_{i_{1}}, \ldots, \sigma_{i_{\rho}}\right)$ associated to minimal bios for $\mathcal{X}$ is called the canonical structure at infinity of $\mathcal{X}$. Correspondingly, the $\rho$-tuple $\left(\varepsilon_{j_{1}}, \ldots, \varepsilon_{j_{\rho}}\right)$ is called the canonical essential structure of $\mathcal{X}$. Throughout, unless otherwise stated, the canonical structure at infinity and the canonical essential structure of a given system $\mathcal{X}$ will be denoted by $\sigma^{\mathrm{c}}(\mathcal{X})=$ $\left(\sigma_{1}^{\mathrm{c}}, \ldots, \sigma_{\rho}^{\mathrm{c}}\right), \varepsilon^{\mathrm{c}}(\mathcal{X})=\left(\varepsilon_{1}^{\mathrm{c}}, \ldots, \varepsilon_{\rho}^{\mathrm{c}}\right)$, respectively; when the considered system is clearly designated then the mention of $\mathcal{X}$ will be omitted.

Lemma 10. For any system the canonical structure at infinity and the canonical essential structure both consist of rational integers. Moreover, $\sigma_{i}^{\mathrm{c}} \leq \varepsilon_{i}^{\mathrm{c}}(1 \leq i \leq \rho)$, and $\left(\sigma_{1}^{\mathrm{c}}, \ldots, \sigma_{\rho}^{\mathrm{c}}\right)$ is increasing.

Proof. Omitted for lack of space.

The set of minimal bios of system (2) is

$$
\left(\left(u_{1}, u_{2}\right),(),\left(y_{2}, y_{1}\right),()\right), \quad\left(\left(u_{2}, u_{1}\right),(),\left(y_{2}, y_{1}\right),()\right) .
$$

And the canonical structure at infinity is $(0,2)$. The differential minimal polynomials corresponding to the above two bios are

$$
\left\{\begin{array}{l}
\ddot{u}_{1}-\ddot{u}_{2}-u_{2}+\ddot{y}_{2}+y_{2}=0 \\
u_{2}-\ddot{y}_{1}-y_{2}=0
\end{array}\right.
$$

and

$$
\left\{\begin{array}{l}
u_{1}-\ddot{y}_{1}-y_{1}=0, \\
\ddot{u}_{1}-\ddot{u}_{2}-u_{2}+\ddot{y}_{2}+y_{2}=0,
\end{array}\right.
$$

respectively. The canonical essential structure is also $(0,2)$, it is directly read on the above differential minimal polynomials.

\section{Among the ordered bios}

$$
\begin{aligned}
& \left(\left(u_{3}, u_{1}\right),\left(u_{2}\right),\left(y_{1}, y_{2}\right),()\right), \quad\left(\left(u_{1}, u_{2}\right),\left(u_{3}\right),\left(y_{2}, y_{1}\right),()\right), \\
& \left(\left(u_{3}, u_{1}\right),\left(u_{2}\right),\left(y_{2}, y_{1}\right),()\right),
\end{aligned}
$$

of system (4) only

$$
\left(\left(u_{1}, u_{2}\right),\left(u_{3}\right),\left(y_{2}, y_{1}\right),()\right)
$$

is minimal. The differential minimal polynomials are

$$
\left\{\begin{array}{l}
\ddot{u}_{1}-\ddot{y}_{2}=0, \\
u_{2}^{(3)}+\ddot{u}_{3}-y_{1}^{(4)}+\ddot{y}_{2}-\ddot{y}_{1}=0,
\end{array}\right.
$$

The canonical structure at infinity is $(0,1)$, and the canonical essential structure is $(0,1)$.

Let $\varpi$ be a symbol not in $\mathbb{Z}$, and different from $\infty$ and $-\infty$.

Definition 11. Given a minimal bio, $\mathfrak{B}$, of $\mathcal{X}$, the $m$-tuple $\left(\sigma_{1}, \ldots, \sigma_{m}\right)$, where $\sigma_{i_{l}}=\varpi$, for $\rho+1 \leq l \leq m$, is said to be the structure at infinity of $\mathcal{X}$ relatively to $\mathfrak{B}$. An $m$-tuple $\left(\sigma_{1}, \ldots, \sigma_{m}\right)$ is said to be $a$ structure at infinity of $\mathcal{X}$ if it is the structure at infinity of $\mathcal{X}$ relatively to some minimal bio. The structure at infinity of $\mathcal{X}$ is the collection of all the structures at infinity relatively to minimal bios of $\mathcal{X}$. Given a structure at infinity $\left(\sigma_{1}, \ldots, \sigma_{m}\right)$, any input component with index $i$ such that $\sigma_{i}=\varpi$ is referred to as a $\varpi$-input. The $p$-tuple $\left(\varepsilon_{1}, \ldots, \varepsilon_{p}\right)$, where $\varepsilon_{j_{l}}=\varpi$, for $\rho+1 \leq l \leq p$, is said to be the essential structure of $\mathcal{X}$ relatively to $\mathfrak{B}$. A $p$-tuple $\left(\varepsilon_{1}, \ldots, \varepsilon_{p}\right)$ is said to be an essential structure of $\mathcal{X}$ if it is the essential structure of $\mathcal{X}$ relatively to some minimal bio. The essential structure of $\mathcal{X}$ is the collection of all the essential structures relatively to minimal bios of $\mathcal{X}$. Given an essential structure $\left(\varepsilon_{1}, \ldots, \varepsilon_{p}\right)$, any output component with index $i$ such that $\varepsilon_{i}=\varpi$ is referred to as a $\varpi$-output.

System (4) illustrates enough the main point in defining the structure at infinity as tuples of $m$ elements. It was seen that the only minimal bio for system (4) is $\left(\left(u_{1}, u_{2}\right),\left(u_{3}\right),\left(y_{2}, y_{1}\right),()\right)$, and the canonical structure at infinity is $(0,1)$. The structure at infinity is then $(0,1, \varpi)$. 0 corresponds to $u_{1}, 1$ to $u_{2}$ and $\varpi$ to $u_{3}$. Moreover, there is no other way, for this system, to make such a correspondence between the structure at infinity and the input components. This definite correspondence is made available thanks to the introduction of $\varpi$. The essential structure of the same system is $(1,0)$. Again, 1 corresponds to $y_{1}$ and 0 to $y_{2}$.

Remark 12. The fact that the structure at infinity is better defined as a collection of tuples rather than only one tuple as in the classical approach is suggested for example by the system (2), since its structure at infinity is given by the following two couples $(0,2)$, and $(2,0)$ corresponding to the respective two minimal bios of system (2).

\section{COMPUTING}

One of the major points of the new definitions of the structure at infinity and the essential structure is that these quantities are computable for, virtually, any given algebraic system, provided that characteristic sets are constructive over the differential field $\mathbf{k}$. However it should be clear that the computations involved by the structures of a system are intrinsically tedious. No doubt that there always will be examples of system the computation of whose structures at infinity will overflow any available computer, no matter how powerful that machine is. The challenge is to obtain devices which bypass unnecessary computations. This section produces preliminary results towards that goal.

Characteristic set computations of $\rho$ are first considered. Since the defining differential ideal, $\mathbf{I}(\mathcal{X})$, of the system $\mathcal{X}$ is prime in $\mathbf{k}\{U, Z, Y\}$ let $\mathcal{A}$ be a characteristic set of $\mathcal{X}$ for a ranking such that all derivatives of $U$, and $Z$ are lower than $Y$. Then $\mathcal{A}_{y}=\mathcal{A} \cap \mathbf{k}\{Y\}$ is a characteristic set 
of $\mathbf{I}(\mathcal{X}) \cap \mathbf{k}\{Y\}$ Let $Y^{1}$ be the set of $Y^{\prime}$ 's whose derivatives do not appear as leaders of elements of $\mathcal{A}_{y}$. The cardinal of $Y^{1}$ is equal to $\partial_{\mathbf{k}}^{\circ} \mathbf{k}\langle y\rangle$.

In terms of complexity, characteristic set computations with respect to orderly rankings are less expensive than those with respect to so-called elimination rankings (which rank the variables being eliminated higher than any derivative of the other variables). Therefore, the main point of Lemma 5 is to compute $\rho$ from $\partial_{\mathbf{k}\langle y\rangle}^{\circ} \mathbf{k}\langle u, z, y\rangle$ which is the number of variables whose derivatives do not appear as leaders of polynomials in the characteristic set of the extension of $\mathbf{I}(\mathcal{X})$ to $\mathbf{k}\langle Y\rangle\{U, Z\}$ with respect to orderly rankings such that all derivatives of $Z$ are lower than $U$.

Let $o_{\tau_{i}}(R)$ be the order in $T_{i}$ of a given differential polynomial $R$ in the differential polynomial algebra $\mathbf{k}\{T\}=$ $\mathbf{k}\left\{T_{1}, \ldots, T_{\mu}\right\}$. Let $\mathrm{d}_{\tau_{i}}^{\circ}(R)$ be the degree of $R$ in $T_{i}^{\left(o_{\tau_{i}}(R)\right)}$ for a differential polynomial $R$ with nonnegative order in $T_{i}$, and let $\mathrm{d}_{\tau_{i}}^{\circ}(R)$ be $-\infty$ when $R$ does not involve $T_{i}$. Let $\omega_{\tau_{i}}(R)$ be the couple $\left(o_{\tau_{i}}(R), \mathrm{d}_{\tau_{i}}^{\circ}(R)\right)$. Let these couples be ordered lexicographically. When the context makes it clear the $\tau$ in the subindexes will be dropped, in particular, $\omega_{\mu}$ will stand for $\omega_{\tau_{\mu}}$.

Let $\mathbf{k}\left\langle\tau_{1}, \ldots, \tau_{\mu}\right\rangle$ be a differential field extension of $\mathbf{k}$ with $\left(\tau_{1}, \ldots, \tau_{\mu^{\prime}}\right)$ as a differential transcendence base, where $\mu^{\prime}<\mu-1$. An element $P(T)$ of $\mathbf{k}\{T\}$ is said to be $a$ minimal polynomial of $\tau_{\mu}$ over $\mathbf{k}\left\langle\tau_{1}, \ldots, \tau_{\mu-1}\right\rangle$ if

(i) $P(T)$ involves $T_{\mu}$,

(ii) $P(\tau)=0$,

(iii) $P(T)$ is k-irreducible,

(iv) $P(T)$ is with lowest $\omega_{\mu}$.

There is a differential minimal polynomial for any $\tau_{i}$, $\mu^{\prime}+1 \leq i \leq \mu$, over $\mathbf{k}\left\langle\tau_{1}, \ldots, \tau_{i-1}\right\rangle$ since $\left(\tau_{1}, \ldots, \tau_{\mu^{\prime}}\right)$ is a differential transcendence base of $\mathbf{k}\left\langle\tau_{1}, \ldots, \tau_{\mu}\right\rangle$ over $\mathbf{k}$. But, as noted earlier, such polynomials are not uniquely determined.

Let $T_{\mu-1}$ be present in $P$. An element $Q(T)$ of $\mathbf{k}\{T\}$ is said to be a minimal eliminating polynomial of $\tau_{\mu-1}$ in $P$ over $\mathbf{k}\left\langle\tau_{1}, \tau_{2}, \ldots, \tau_{\mu-2}, \tau_{\mu}\right\rangle$ if it is with lowest $\omega_{\mu-1}$ in the subset of $\mathbf{k}\{T\}$ consisting of differential polynomials $R$ such that

(i) $R$ involves $T_{\mu-1}$

(ii) $R(\tau)=0$,

(iii) $R$ is $\mathbf{k}$-irreducible,

(iv) $R(P$, respectively $)$ is not contained in the differential ideal generated by $P$ ( $R$, respectively).

There always is a minimal eliminating polynomial of $\tau_{\mu-1}$ in $P$ over the differential field $\mathbf{k}\left\langle\tau_{1}, \tau_{2}, \ldots, \tau_{\mu-2}, \tau_{\mu}\right\rangle$. It is sufficient to take a differential minimal polynomial of $\tau_{\mu-1}$ over $\mathbf{k}\left\langle\tau_{1}, \tau_{2}, \ldots, \tau_{\mu-2}\right\rangle$. The only point is that such a strategy may be more expensive in computations.

Lemma 13. Let $\mathbf{k}\left\langle\tau_{1}, \ldots, \tau_{\mu}\right\rangle$ be a differential field extension of $\mathbf{k}$ such that $\left(\tau_{1}, \tau_{2}, \ldots, \tau_{\mu^{\prime}}\right)$ is a differential transcendence base, where $\mu^{\prime}<\mu-1$. Let $P(T)$ be a differential minimal polynomial of $\tau_{\mu}$ over $\mathbf{k}\left\langle\tau_{1}, \ldots, \tau_{\mu-1}\right\rangle$. Let $T_{\mu-1}$ be present in $P$. Let $Q(T)$ be a minimal eliminating polynomial of $\tau_{\mu-1}$ in $P$. Then there is an element $P^{\prime}$ of $\mathbf{k}\left\{T_{1}, \ldots, T_{\mu-2}, T_{\mu}\right\}$ zeroed by $\tau$, and such that, for any $T_{i}$ among $T_{1}, \ldots, T_{\mu-2}, T_{\mu}$, the condition

$$
\left\{\begin{array}{l}
o_{\mu-1}(P)-o_{i}(P) \neq o_{\mu-1}(Q)-o_{i}(Q) \\
o_{\mu-1}(P) \neq o_{\mu-1}(Q)
\end{array}\right.
$$

implies

$$
\begin{aligned}
& o_{i}\left(P^{\prime}\right)=\max \left(o_{i}(P), o_{i}(P)+\left(o_{\mu-1}(Q)-o_{\mu-1}(P)\right)\right. \\
& \left.o_{i}(Q), o_{i}(Q)+\left(o_{\mu-1}(P)-o_{\mu-1}(Q)\right)\right) .
\end{aligned}
$$

Moreover, if

$$
\left\{\begin{array}{l}
o_{\mu-1}(P)-o_{i}(P)<o_{\mu-1}(Q)-o_{i}(Q), \\
o_{\mu-1}(P)<o_{\mu-1}(Q),
\end{array}\right.
$$

then $P^{\prime}$ is a differential minimal polynomial of $T_{\mu}$ over $\mathbf{k}\left\langle\tau_{1}, \ldots, \tau_{\mu-2}\right\rangle$.

Corollary 14. Let $\mathcal{X}$ be a system with positive differential output rank, $\rho$. Let $\mathfrak{B}$ be a bio of $\mathcal{X}$. Let $l$ be a natural integer, $1 \leq l \leq \rho$. Let

$$
P\left(y_{j_{1}}, \ldots, y_{j_{l}}, u_{i_{l+1}}, \ldots, u_{i_{m}}, \xi, u_{i_{l}}\right)
$$

be a differential minimal polynomial of $u_{i_{l}}$ over the differential field

$$
\mathbf{k}\left\langle y_{j_{1}}, \ldots, y_{j_{l}}, u_{i_{l+1}}, \ldots, u_{i_{m}}, \xi\right\rangle
$$

where $\xi=\left(\xi_{1}, \ldots, \xi_{\mu}\right)$ is some subset of $z_{1}, \ldots, z_{\nu}, y_{j_{l+1}}$, $\ldots, y_{j_{p}}$. Let $\xi_{\mu}$ be one of the components of $\xi$ which are present in $P$. Given a differential polynomial $R$ in $\left(y_{j_{1}}, \ldots, y_{j_{l}}, u_{i_{l}}, \ldots, u_{i_{m}}, \xi\right)$, let $\alpha_{i_{k}}(R)$ be $o_{u_{i_{k}}}(R)$ for $k \in$ $\mathbb{N}, l \leq k \leq m$. Let $\gamma_{\mu}(R)$ be $o_{\xi_{\mu}}(R)$. Let $\beta_{j_{k}}(R)$ be $o_{y_{j_{k}}}(R)$ for $k \in \mathbb{N}, 1 \leq k \leq l$. Let $Q\left(y_{j_{1}}, \ldots, y_{j_{l}}, u_{i_{l+1}}, \ldots, u_{i_{m}}, \xi\right.$, $u_{i_{l}}$ ) be a minimal eliminating polynomial of $\xi_{\mu}$ in $P$. Then $u_{i_{l}}$ satisfies an equation, $P^{\prime}=0$, over

$$
\mathbf{k}\left\langle y_{j_{1}}, \ldots, y_{j_{l}}, u_{i_{l+1}}, \ldots, u_{i_{m}}, \xi_{1}, \ldots, \xi_{\mu-1}\right\rangle .
$$

If $\gamma_{\mu}(P) \neq \gamma_{\mu}(Q)$ then, for each $k, k^{\prime} \in \mathbb{N}, l \leq k \leq m, 1 \leq$ $k^{\prime} \leq l$,

$$
\left\{\begin{array}{l}
\gamma_{\mu}(P)-\alpha_{i_{k}}(P)<\gamma_{\mu}(Q)-\alpha_{i_{k}}(Q) \\
\beta_{j_{k^{\prime}}}(P)-\gamma_{\mu}(P)>\beta_{j_{k^{\prime}}}(Q)-\gamma_{\mu}(Q)
\end{array}\right.
$$

$$
\Downarrow
$$

$$
\beta_{j_{k^{\prime}}}\left(P^{\prime}\right)-\alpha_{i_{k}}\left(P^{\prime}\right)=\beta_{j_{k^{\prime}}}(P)-\alpha_{i_{k}}(P),
$$

and

$$
\left\{\begin{array}{l}
\gamma_{\mu}(P)-\alpha_{i_{k}}(P)<\gamma_{\mu}(Q)-\alpha_{i_{k}}(Q), \\
\beta_{j_{k^{\prime}}}(P)-\gamma_{\mu}(P)<\beta_{j_{k^{\prime}}}(Q)-\gamma_{\mu}(Q)
\end{array}\right.
$$

$\Downarrow$

and

$$
\begin{gathered}
\beta_{j_{k^{\prime}}}\left(P^{\prime}\right)-\alpha_{i_{k}}\left(P^{\prime}\right)= \\
\left(\gamma_{\mu}(P)-\alpha_{i_{k}}(P)\right)+\left(\beta_{j_{k^{\prime}}}(Q)-\gamma_{\mu}(Q)\right),
\end{gathered}
$$$$
\left\{\begin{array}{l}
\gamma_{\mu}(P)-\alpha_{i_{k}}(P)>\gamma_{\mu}(Q)-\alpha_{i_{k}}(Q), \\
\beta_{j_{k^{\prime}}}(P)-\gamma_{\mu}(P)<\beta_{j_{k^{\prime}}}(Q)-\gamma_{\mu}(Q)
\end{array}\right.
$$$$
\Downarrow
$$

and

$$
\beta_{j_{k^{\prime}}}\left(P^{\prime}\right)-\alpha_{i_{k}}\left(P^{\prime}\right)=\beta_{j_{k^{\prime}}}(Q)-\alpha_{i_{k}}(Q),
$$

$$
\begin{gathered}
\left\{\begin{array}{c}
\gamma_{\mu}(P)-\alpha_{i_{k}}(P)>\gamma_{\mu}(Q)-\alpha_{i_{k}}(Q), \\
\beta_{j_{k^{\prime}}}(P)-\gamma_{\mu}(P)>\beta_{j_{k^{\prime}}}(Q)-\gamma_{\mu}(Q)
\end{array}\right. \\
\Downarrow \\
\left(\beta_{j_{k^{\prime}}}(P)-\gamma_{\mu}(P)\right)+\left(\gamma_{\mu}(Q)-\alpha_{i_{k}}(Q)\right) .
\end{gathered}
$$


If

$$
\left\{\begin{array}{l}
\gamma_{\mu}(P)-\alpha_{i_{l}}(P)<\gamma_{\mu}(Q)-\alpha_{i_{l}}(Q), \\
\gamma_{\mu}(P) \neq \gamma_{\mu}(Q),
\end{array}\right.
$$

then, over $\mathbf{k}\left\langle y_{j_{1}}, \ldots, y_{j_{l}}, u_{i_{l+1}}, \ldots, u_{i_{m}}, \xi_{1}, \ldots, \xi_{\mu-1}\right\rangle, P^{\prime}$ is a differential minimal polynomial of $u_{i_{l}}$.

This is obtained by merely inspecting formula (5) under the different cases listed in the corollary.

Corollary 15. Let $\mathcal{X}$ be a system with positive differential output rank, $\rho$. Let $\mathfrak{B}$ be a bio of $\mathcal{X}$. Let $l$ be a natural integer such that $1 \leq l \leq \rho$. Let $P\left(y_{j_{1}}, \ldots, y_{j_{l}}, u_{i_{l+1}}, \ldots, u_{i_{m}}, \xi, u_{i_{l}}\right)$ be a differential minimal polynomial of $u_{i_{l}}$ over $\mathbf{k}\left\langle y_{j_{1}}, \ldots, y_{j_{l}}, u_{i_{l+1}}, \ldots, u_{i_{m}}, \xi\right\rangle$ where $\xi=\left(\xi_{1}, \ldots, \xi_{\mu}\right)$ is some subset of $z_{1}, \ldots, z_{\nu}, y_{j_{l+1}}$, $\ldots, y_{j_{p}}$. Let all the $\xi$ 's be present in $P$. For $i \in \mathbb{N}$, $1 \leq i \leq \mu$, let $Q_{i}\left(y_{j_{1}}, \ldots, y_{j_{l}}, u_{i_{l+1}}, \ldots, u_{i_{m}}, \xi, u_{i_{l}}\right)$ be a minimal eliminating polynomial of $\xi_{i}$ in $P$. If, for each $i, \quad \gamma_{i}(P)-\alpha_{i_{k}}(P)<\gamma_{i}\left(Q_{i}\right)-\alpha_{i_{k}}\left(Q_{i}\right)$ and $\beta_{j_{k^{\prime}}}(P)-$ $\gamma_{i}(P)>\beta_{j_{k^{\prime}}}\left(Q_{i}\right)-\gamma_{i}\left(Q_{i}\right)$ then $u_{i_{l}}$ satisfies a minimal equation, $P^{\prime}=0$, over $\mathbf{k}\left\langle y_{j_{1}}, \ldots, y_{j_{l}}, u_{i_{l+1}}, \ldots, u_{i_{m}}\right\rangle$ such that, for each $k, k^{\prime} \in \mathbb{N}, l \leq k \leq m, 1 \leq k^{\prime} \leq l, \beta_{j_{k^{\prime}}}\left(P^{\prime}\right)-$ $\alpha_{i_{k}}\left(P^{\prime}\right)=\beta_{j_{k^{\prime}}}(P)-\alpha_{i_{k}}(P)$.

The elimination of the $\xi$ 's may be conducted one by one. The corollary then immediately results from Corollary 14 and Lemma 13.

Corollary 16. Let $\mathcal{X}$ be a system with positive differential output rank, $\rho$. Let $\mathfrak{B}$ be a bio of $\mathcal{X}$. Let there exist relations

$$
\left\{\begin{array}{l}
P_{1}\left(y_{j_{1}}, u_{i_{2}}, \ldots, u_{i_{m}}, \xi^{1}, u_{i_{1}}\right)=0 \\
P_{2}\left(y_{j_{1}}, y_{j_{2}}, u_{i_{3}}, \ldots, u_{i_{m}}, \xi^{2}, u_{i_{2}}\right)=0 \\
\ldots \\
P_{\rho-1}\left(y_{j_{1}}, \ldots, y_{j_{\rho-1}}, u_{i_{\rho}}, \ldots, u_{i_{m}}, \xi^{\rho-1}, u_{i_{\rho-1}}\right)=0 \\
P_{\rho}\left(y_{j_{1}}, \ldots, y_{j_{\rho}}, u_{i_{\rho+1}}, \ldots, u_{i_{m}}, \xi^{\rho}, u_{i_{\rho}}\right)=0
\end{array}\right.
$$

where $\xi^{l}, 1 \leq l \leq \rho$, denotes a subset of $z_{1}, \ldots, z_{\nu}, y_{j_{l+1}}$, $\ldots, y_{j_{p}}$ such that $P_{l}$ is a differential minimal polynomial of $u_{i_{l}}$ over $\mathbf{k}\left\langle y_{j_{1}}, \ldots, y_{j_{l}}, u_{i_{l+1}}, \ldots, u_{i_{m}}, \xi^{l}, u_{i_{l}}\right\rangle$. Let all the $\xi_{i}^{l}$ 's in $\xi^{l}=\left(\xi_{1}^{l}, \ldots, \xi_{\mu_{l}}^{l}\right)$ be present in $P_{l}$. For $i, 1 \leq i \leq$ $\mu_{l}$, let $Q_{i}^{l}\left(y_{j_{1}}, \ldots, y_{j_{l}}, u_{i_{l+1}}, \ldots, u_{i_{m}}, \xi^{l}, u_{i_{l}}\right)$ be a minimal eliminating polynomial of $\xi_{i}^{l}$ in $P_{l}$. If, for each $l, i$,

$$
\left\{\begin{array}{l}
\gamma_{i}^{l}\left(P_{l}\right)-\alpha_{i_{k}}\left(P_{l}\right)<\gamma_{i}^{l}\left(Q_{i}^{l}\right)-\alpha_{i_{k}}\left(Q_{i}^{l}\right), \\
\beta_{j_{k^{\prime}}}\left(P_{l}\right)-\gamma_{i}^{l}\left(P_{l}\right)>\beta_{j_{k^{\prime}}}\left(Q_{i}^{l}\right)-\gamma_{i}^{l}\left(Q_{i}^{l}\right),
\end{array}\right.
$$

then there are relations

$$
\left\{\begin{array}{l}
P_{1}^{\prime}\left(y_{j_{1}}, u_{i_{2}}, \ldots, u_{i_{m}}, u_{i_{1}}\right)=0 \\
P_{2}^{\prime}\left(y_{j_{1}}, y_{j_{2}}, u_{i_{3}}, \ldots, u_{i_{m}}, u_{i_{2}}\right)=0 \\
\ldots \\
P_{\rho-1}^{\prime}\left(y_{j_{1}}, \ldots, y_{j_{\rho-1}}, u_{i_{\rho}}, \ldots, u_{i_{m}}, u_{i_{\rho-1}}\right)=0 \\
P_{\rho}^{\prime}\left(y_{j_{1}}, \ldots, y_{j_{\rho}}, u_{i_{\rho+1}}, \ldots, u_{i_{m}}, u_{i_{\rho}}\right)=0
\end{array}\right.
$$

such that $P_{l}^{\prime}$ is a differential minimal polynomial of $u_{i_{l}}$ over the differential field $\mathbf{k}\left\langle y_{j_{1}}, \ldots, y_{j_{l}}, u_{i_{l+1}}, \ldots, u_{i_{m}}\right\rangle$, $\beta_{j_{k^{\prime}}}\left(P_{l}^{\prime}\right)-\alpha_{i_{k}}\left(P_{l}^{\prime}\right)=\beta_{j_{k^{\prime}}}\left(P_{l}\right)-\alpha_{i_{k}}\left(P_{l}\right)$ for each $l, k, k^{\prime} \in$ $\mathbb{N}, 1 \leq l \leq \rho, l \leq k \leq m, 1 \leq k^{\prime} \leq l$.

This is again an easy consequence of the previous Lemma and Corollaries.

The latter result makes possible to avoid the computation of the differential minimal polynomials (8) as suggested by Definition 8. Instead, the information on the structure at infinity and essential structure is directly extracted from relations of type (7) which, typically, are easier to derive.

\section{COMPARING WITH THE CLASSICAL APPROACH}

It is shown how the classical list of integers which stands for the structure at infinity may be recovered from the new structure at infinity.

The following definite link between the new structure at infinity and the classical one is thus proved.

Theorem 17. For systems of type (1) the sequence of integers, $\rho_{1}, \ldots, \rho_{n}$, of the classical structure algorithm is obtained from the canonical structure at infinity, $\sigma^{\mathrm{c}}=$ $\left(\sigma_{1}^{\mathrm{c}}, \ldots, \sigma_{\rho}^{\mathrm{c}}\right)$, as follows: $\rho_{i}$ is the number of elements of $\sigma^{\mathrm{c}}$ which are less than or equal to $i$.

\section{REFERENCES}

Aït Amirat, Y. (1994). Contribution à la Théorie de la Structure des Systèmes en Automatique. Application au Découplage et au Rejet de Perturbations. Thesis, Université Claude Bernard Lyon I, Villeurbanne, France.

Belur, M.N. (2011). Impulsive solutions, inadmissible initial conditions and pole/zero structure at infinity. In Proceedings of the IEEE Conference on Decision and Control, 1528-1533. IEEE Press, New York. doi: 10.1109/CDC.2011.6161440.

di Benedetto, M.D., Grizzle, J.W., and Moog, C.H. (1989). Rank invariants of nonlinear systems. SIAM J. Control Optim., 27, 658-672.

Diop, S. (2002). From the geometry to the algebra of nonlinear observability. In A. Anzaldo-Meneses, B. Bonnard, J.P. Gauthier, and F. Monroy-Perez (eds.), Contemporary Trends in Nonlinear Geometric Control Theory and its Applications, 305-345. World Scientific Publishing Co., Singapore.

Fliess, M. (1989). Automatique et corps différentiels. Forum Math., 1, 227-238.

Kolchin, E.R. (1973). Differential Algebra and Algebraic Groups. Academic Press, New York.

Laakkonen, P. and Pohjolainen, S. (2011). Directed structure at infinity for infinite-dimensional systems. Internat. J. Control, 84, 702-715. doi: 10.1080/00207179.2011.572999.

Pereira da Silva, P.S., Veloso Pazzoto, N.A., and Correa Filho, C. (2006). Relative structure at infnity and nonlinear semi-implicit daes. European J. Control, 12, 545-558. doi:10.3166/ejc.12.545-558. 